Proyecciones Journal of Mathematics Vol. 34, $\mathrm{N}^{o}$ 1, pp. 69-83, March 2015.

Universidad Católica del Norte

Antofagasta - Chile

\title{
State analysis of time-varying singular nonlinear systems using Legendre wavelets
}

\author{
S. Raja Balachandar \\ S. G. Venkatesh \\ S. K. Ayyaswamy \\ and \\ S. Balachandran \\ Sastra University, India \\ Received : December 2014. Accepted : March 2015
}

\begin{abstract}
In this paper, the Legendre wavelet method for State analysis of time-varying singular nonlinear systems is studied. The properties of Legendre wavelets and its operational matrices are first presented and then are used to convert into algebraic equations. Also the convergence and error analysis for the proposed technique have been discussed. Illustrative examples have been given to demonstrate the validity and applicability of the technique. The efficiency of the proposed method has been compared with Haar wavelet method and it is observed that the Legendre wavelet method is more convenient than the Haar wavelet method in terms of applicability, efficiency, accuracy, error, and computational effort.
\end{abstract}

Keywords : Legendre wavelets; Time-varying; Singular nonlinear systems; Convergence analysis; Operational matrix.

AMS Subject Classification: 34B05, 47E99 


\section{Introduction}

The development of singular nonlinear systems has been studied by Newcomb and campbell $[1,2]$. In their papers, closed form solutions were not available. In some analysis of neural networks, both singular systems [3] and nonlinear systems [4] have been used. The multipliers and algebraic interconnections between singular systems and nonlinear systems are allowed in dynamical systems. Lewis et al. [5] studied singular bilinear systems extensively in the literature. However, the solution due to Lewis et al. only applies for the time-invariant case.

Recently, Haar wavelets have been applied extensively for signal processing in communications and physics research, and proved to be a useful mathematical tool. Chen and Hsiao [6] introduced Haar operational matrix for the integrals of Haar functions and this approach was also applied to system analysis and dynamical systems. Later, Hsiao [7] developed the Haar product matrix and coefficient matrix which have been successfully implemented to the solution and the optimization of time-varying systems [8]. The main characteristic of this technique is to convert a differential equation into an algebraic one; hence the solution and the optimization procedures are either reduced or simplified accordingly.

Sekar et al. [9] handled Haar wavelet series method for higher order linear singular system. Murugesh et al. [10] discussed State analysis of timeVarying singular bilinear systems using RK-Butcher Algorithms. Sepehrian et al. [11] demonstrated the solution of time-varying singular nonlinear systems by using single term walsh series. A Generalized approach for state analysis and parameter estimation of bilinear systems using Haar connection coefficients was studied by Garg et al. [12]. Pin-Lin Liu [13] adopted a delay decomposition approach on stability analysis for singular systems with time varying delay. Henrik Sandberg [14] investigated a balanced truncation of linear time-varying systems in discrete and continuous time. Recently, Zhou et al. [15] studied finite-time stability analysis for linear time-varying singular impulsive systems.

Mirmomeni [16] established fuzzy neural network approach for state analysis of discrete-time singular nonlinear Systems. A new method for simplification of nonlinear input-output models was outlined by Nilsson et al. [17]. The method relates to balanced truncation and uses a state transformation followed by truncation of some states. Timescale analysis for nonlinear dynamical systems and robust stability and stabilization of 
discrete singular systems were also studied in [18]. Very recently, Berstein polynomial operational matrix approach was introduced by Behroozifar et al. [19] for numerical solution of optimal control of time-varying singular systems. Balachandran et al. [20] used the single term Walsh series (STWS) technique for the analysis of electronic circuits. They have also analyzed singular system of transistor circuits by STWS technique in [21].

In this paper, we apply the Legendre wavelets operational matrix of integration for state analysis of time-varying singular nonlinear systems. Legendre wavelets are capable of converting the given system of differential equations into system of algebraic equations. Many researchers have employed the Legendre wavelets using operational matrix of integration and with the help of Gaussian integration. The detailed information are available in [22-33].

The arrangement of the paper is as follows: In section 2, we describe the basic formulation of wavelets and Legendre wavelets required for our subsequent development. Section 3 is devoted to the state analysis of timevarying singular nonlinear systems. Convergence analysis and the error bound for the proposed method have been discussed in section 4 . In section 5 , we demonstrate the accuracy of the proposed scheme by considering numerical examples. Concluding remarks are given in the final section.

\section{Properties of Legendre wavelets}

\subsection{Wavelets and Legendre wavelets}

Wavelets constitute a family of functions constructed from dilation and translation of a single function called the mother wavelet. When the dilation parameter 'a' and the translation parameter 'b' vary continuously, we have the following family of continuous wavelets as:

$$
\psi_{a, b}(t)=|a|^{-\frac{1}{2}} \psi\left(\frac{t-b}{a}\right), a, b \in R, a \neq 0
$$

If we restrict the parameters 'a' and 'b' to discrete values as $a=a_{0}^{-k}$, $b=n b_{0} a_{0}^{-k}, a_{0}>1, b_{0}>0$ and $\mathrm{n}, \mathrm{k}$ are positive integers, we have the following family of discrete wavelets: $\psi_{k, n}(t)=|a|^{-\frac{1}{2}} \psi\left(\left(a_{0}\right)^{k} t-n b_{0}\right)$ where $\psi_{k, n}(t)$ forms an orthonormal basis.

Legendre wavelets $\psi_{n, m}(t)=\psi(k, \hat{n}, m, t)$ have four arguements: $\hat{n}=$ $2 n-1, n=1,2,3, \ldots, 2^{k-1}, \mathrm{k}$ can assume any positive integer, $\mathrm{m}$ is the order of Legendre polynomials and $t$ is the normalized time. They are defined on the interval $[0,1)$ as 


$$
\psi_{n, m}(t)=\left\{\begin{array}{cl}
\sqrt{m+\frac{1}{2}} 2^{\frac{k}{2}} P_{m}\left(2^{k} t-\hat{n}\right) & , \text { for } \frac{\hat{n}-1}{2^{k}} \leq t \leq \frac{\hat{n}+1}{2^{k}} \\
0 & , \text { otherwise }
\end{array}\right\}
$$

where $m=0,1,2, \ldots, M-1, n=1,2,3, \ldots, 2^{k-1}$. The coefficient $\sqrt{m+\frac{1}{2}}$ is for orthonormality, the dilation parameter is $a=2^{-k}$ and translation parameter is $b=\hat{n} 2^{-k}$. Here $P_{m}(t)$ are well-known Legendre polynomials of order $m$ which are defined on the interval $[-1,1]$, and can be determined with the aid of the following recurrence formulae:

$$
P_{0}(t)=1, P_{1}(t)=t, P_{m+1}(t)=\left(\frac{2 m+1}{m+1}\right) t P_{m}(t)-\left(\frac{m}{m+1}\right) P_{m-1}(t),
$$
where $\mathrm{m}=1,2,3, \ldots$

\subsection{Function Approximation}

A function $u(x, t)$ defined over $[0,1)$ may be expanded as

$$
f(t)=\sum_{n=1}^{\infty} \sum_{m=0}^{\infty} c_{n m} \psi_{n m}(t)
$$

where $c_{n m}=\left\langle f(t), \psi_{n m}(t)\right\rangle$, in which $\langle.,$.$\rangle denotes the inner product.$

If the infinite series in Eq.(2.2) is truncated, then Eq.(2.2) can be written as

$$
f(t)=\sum_{n=1}^{2^{k-1}} \sum_{m=0}^{M-1} c_{n m} \psi_{n m}(t)=C^{T} \psi(t)
$$

where $\mathrm{C}$ and $\psi(t)$ are $2^{k-1} M \times 1$ matrices given by

$$
C=\left[c_{10}, c_{11}, \cdots, c_{1 M-1}, c_{20}, c_{21}, \cdots, c_{2 M-1}, \cdots, c_{2^{K-1} 0}, \cdots, c_{2^{K-1} M-1}\right]^{T}
$$

$$
\begin{aligned}
& \psi(t)=\left[\psi_{10}(t), \cdots, \psi_{1 M-1}(t), \psi_{20}(t), \cdots, \psi_{2 M-1}(t), \cdots, \psi_{2^{K-1} 0}(t), \cdots,\right. \\
& \left.\psi_{2^{K-1} M-1}(t)\right]^{T}
\end{aligned}
$$


The integration of vector $\psi(t)$ defined in Eq. (2.3) is given by $\int_{0}^{T} \psi\left(t^{\prime}\right) d t^{\prime}=P \psi(t)$ where $\mathrm{P}$ is $\left(2^{k-1} M\right) X\left(2^{k-1} M\right)$ operational matrix of integration and is given in [19] as

$$
P=\frac{1}{2^{k}}\left[\begin{array}{ccccc}
L & F & F & \cdots & F \\
O & L & F & \cdots & F \\
\vdots & \vdots & \vdots & \vdots & \vdots \\
O & O & \cdots & O & L
\end{array}\right]
$$

In Eq.(2.6) $\mathrm{F}$ and $\mathrm{L}$ are $\mathrm{M}$ x M matrices given by $F=\left[\begin{array}{ccc}2 & \cdots & 0 \\ \vdots & \ddots & \vdots \\ 0 & \cdots & 0\end{array}\right]$ and

$L=\left[\begin{array}{rrrrlrrr}1 & \frac{1}{\sqrt{3}} & 0 & 0 & \cdots & 0 & 0 & 0 \\ \frac{\sqrt{3}}{3} & 0 & \frac{\sqrt{3}}{3 \sqrt{5}} & 0 & \cdots & 0 & 0 & 0 \\ 0 & \frac{\sqrt{5}}{5 \sqrt{3}} & 0 & \frac{\sqrt{5}}{5 \sqrt{7}} & \cdots & 0 & 0 & 0 \\ 0 & 0 & \frac{\sqrt{7}}{7 \sqrt{5}} & 0 & \cdots & 0 & 0 & 0 \\ \vdots & \vdots & \vdots & \vdots & \cdots & \vdots & \vdots & \vdots \\ 0 & 0 & 0 & 0 & \cdots & \frac{\sqrt{2 M-3}}{(2 M-3) \sqrt{2 M-5}} & 0 & \frac{\sqrt{2 M-3}}{(2 M-3) \sqrt{2 M-1}} \\ 0 & 0 & 0 & 0 & \cdots & 0 & \frac{\sqrt{2 M-1}}{(2 M-1) \sqrt{2 M-3}} & 0\end{array}\right]$

The integration of the product of two Legendre wavelet function vectors is obtained as $I=\int_{0}^{1} \psi(t) \psi^{T}(t) d t$ where $\mathrm{I}$ is an identity matrix.

\section{State analysis of time-varying singular nonlinear systems using Legendre wavelets}

Consider a time-varying singular nonlinear system of the following form:

$$
E(t) \dot{X}(t)=f(t, x(t), u(t)), x(0)=x_{0}
$$

where

$$
f(t, x(t), u(t))=H(t) x(t)+U(t)
$$


74 S. Raja B., S. G. Venkatesh, S. K. Ayyaswamy \& S. Balachandran

where the singular matrix $\mathrm{E}(\mathrm{t}) \in R^{n \times n}$, the nonlinear function $\mathrm{f} \in R^{n}$, the state variable $\mathrm{x}(\mathrm{t}) \in R^{n}$, the control variable $\mathrm{u}(\mathrm{t}) \in R^{q}$. The response $\mathrm{x}(\mathrm{t})$, $0 \leq t \leq t_{f}$, is required to be found.

Let $\dot{X}(t)=F \psi(t) ; E(t)=e \psi(t) ; H(t)=h \psi(t) ; U(t)=u \psi(t)$

This implies $X(t)=\int_{0}^{t} F \psi(t) d t$

$X(t)=F P \psi(t)+X_{0}[17]$

Eq.(3.1) takes the form $E(t) \dot{X}(t)=H(t) x(t)+U(t)$, becomes

e $\psi(t) F \psi(t)=h \psi(t)\left(F P \psi(t)+x_{0}\right)+u \psi(t)$

$[e \psi(t) F-h \psi(t) F P]=\left[\left[x_{0}, 0,0, \cdots, 0\right]+g\right]$

$[e-h P] \psi F=G_{1}$ where $G_{1}=\left[\left[x_{0}, 0,0, \cdots, 0\right]+g\right]$

The unknown matrix can be solved using Kronecker product as $(\psi F)^{T}=\left[e \otimes I-h \otimes P^{T}\right]^{-1} G_{1}^{T}$

i.e $F^{T} \psi^{T}=\left[e \otimes I-h \otimes P^{T}\right]^{-1} G_{1}^{T}$

$F^{T}=\left[e \otimes I-h \otimes P^{T}\right]^{-1} G_{1}^{T}\left(\psi^{-1}\right)^{T}$

where $F^{T}=\left[\begin{array}{c}f_{0} \\ f_{1} \\ \vdots \\ f_{2^{k-1}(M-1)}\end{array}\right]$ and

$G_{1}^{T}=\left[\begin{array}{c}g_{0} \\ g_{1} \\ \vdots \\ g_{2^{k-1}(M-1)}\end{array}\right]$

The Kronecker product $A \otimes P^{T}$ is defined as in [34]. 


\section{Convergence Analysis}

Theorem 1. [26]

The sum $f(t) \cong \sum_{n=1}^{2 k-1} \sum_{m=0}^{M-1} c_{n m} \psi_{n m}(t)=C^{T} \psi(t)$ converges to the solution $\mathrm{x}(\mathrm{t})$ of Equations (3.1) and (3.2).

Theorem 2. [24]

Suppose that the function $f:[0,1] \rightarrow R$ is $m$ times continuously differentiable, $f \in C^{m}[0,1]$, then $C^{T} \psi$ approximate $f$ with mean error bound as

$$
\left\|f-C^{T} \psi\right\| \leq \frac{1}{\left(m ! 2^{m k}\right)} \sup _{x \in[0,1]}\left|f^{(m)}(x)\right|
$$

\section{Illustrative Examples}

This section will illustrate three numerical examples to show the efficiency of Legendre wavelet approach discussed in [35].

\section{Problem 1 :}

Consider a time varying singular nonlinear system:

$$
\begin{aligned}
& {\left[\begin{array}{ccc}
0 & 1 & 0 \\
0 & 0 & t^{2} \\
0 & 0 & 0
\end{array}\right] \dot{x}(t)=\left[\begin{array}{c}
t x_{1}(t)+x_{2}(t) \\
e^{t} x_{1}(t) x_{2}(t) \\
x_{2}(t)\left(x_{1}(t)+x_{3}(t)\right)
\end{array}\right] \mathrm{x}(\mathrm{t})+\left[\begin{array}{c}
0 \\
2 t^{2} e^{-t} \\
0
\end{array}\right]} \\
& \mathrm{x}(0)=\left[\begin{array}{c}
2 \\
0 \\
-2
\end{array}\right]
\end{aligned}
$$

The analytic solution $\mathrm{x}(\mathrm{t})$ for the above problem is given by

$$
\mathrm{x}(\mathrm{t})=\left[\begin{array}{c}
2 e^{-t}-2 t e^{-t} \\
t^{2} e^{-t} \\
-2 e^{-t}+2 t e^{-t}
\end{array}\right]
$$

The comparison between the Legendre wavelet solution and the analytic solution for $t \in[0,4)$ is shown in Fig. 1 which shows that the Legendre wavelet approach gives almost the same solution with the analytic method given in [35]. 
76 S. Raja B., S. G. Venkatesh, S. K. Ayyaswamy \& S. Balachandran

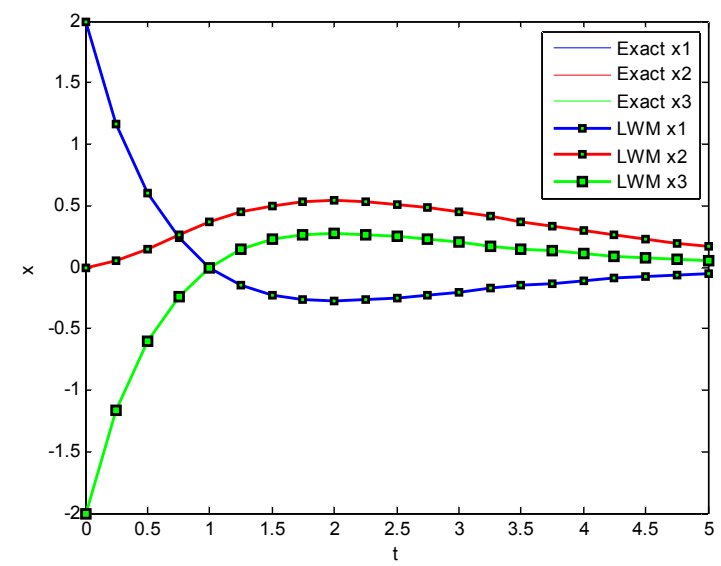

Figure 1 : Comparison between Exact solution and the Legendre wavelet solution for problem 1 .

\section{Problem 2 :}

Consider a time invariant singular nonlinear system defined from the form:

$$
\begin{aligned}
& {\left[\begin{array}{lllll}
1 & 0 & 0 & 0 & 0 \\
0 & 0 & 0 & 0 & 0 \\
0 & 0 & 0 & 0 & 0 \\
0 & 0 & 0 & 0 & 0 \\
0 & 0 & 0 & 0 & 0
\end{array}\right] \dot{x}(t)+\left[\begin{array}{c}
x_{3}(t)-x_{5}(t) \\
x_{2}(t)+x_{3}(t)-x_{4}(t)-x_{5}(t) \\
\left(x_{1}(t)+x_{2}(t)-1\right)^{2}-x_{3}(t) \\
-x_{4}(t) \\
-x_{2}(t)\left(-x_{1}(t)-x_{2}(t)\right)-x_{5}(t)
\end{array}\right]=\left[\begin{array}{l}
0 \\
1 \\
0 \\
0 \\
0
\end{array}\right]} \\
& \mathrm{x}(0)=\left[\begin{array}{l}
0 \\
1 \\
0 \\
0 \\
0
\end{array}\right]
\end{aligned}
$$

The comparison between the Haar wavelet solution and the Legendre wavelet solution is tabulated in Table 5.1.

\section{Problem 3 :}

Consider a time-invariant singular nonlinear system defined from the form: 


$$
\begin{aligned}
& {\left[\begin{array}{lll}
0 & 1 & 0 \\
0 & 0 & 0 \\
0 & 0 & 0
\end{array}\right] \dot{x}(t)+\left[\begin{array}{rrr}
0 & 0 & 1 \\
1 & 1 & 0 \\
1 & 0 & -3
\end{array}\right] x(t)+\left[\begin{array}{c}
0 \\
0 \\
\left(x_{3}\right)^{3}(t)
\end{array}\right]=} \\
& {\left[\begin{array}{l}
0 \\
1 \\
0
\end{array}\right]} \\
& \mathrm{x}(0)=\left[\begin{array}{c}
2 \\
-1 \\
-2
\end{array}\right]
\end{aligned}
$$

The comparison between the Haar wavelet solution and the Legendre wavelet solution is tabulated in Table 5.2. 


\section{Conclusion}

In this paper, Legendre wavelet method for the state analysis of timevarying singular nonlinear systems is studied. The properties of Legendre wavelets and its operational matrices are first presented and then are used to convert into algebraic equations. Also the convergence and error analysis for the proposed technique have been discussed. Illustrative examples have been given to demonstrate the validity and applicability of the technique. The efficiency of the proposed method has been compared with Haar wavelet method and it is observed that the Legendre wavelet method is more convenient than the Haar wavelet method in terms of applicability, efficiency, accuracy, error, and computational effort.

\section{References}

[1] R. W. Newcomb, The semistate description of nonlinear time-variable circuits, IEEE Transactions on Circuits and Systems 28, pp. 62-71, (1981).

[2] S. L. Campbell, Bilinear nonlinear descriptor control systems, CRSC Tech. Rept. 102386-01, Dept. of Math., N.C. State Univ., Raleigh, NC 27695, (1987).

[3] N. Declaris, A. Rindos, Semistate analysis of neural networks in Apysia Californica, Proc. 27th MSCS, pp. 686-689, (1984).

[4] N. Wiener, Cybernetics, MIT Press, Cambridge. MA, (1948).

[5] F. L. Lewis, B. G. Mertzios, W. Marszalek, Analysis of singular bilinear systems using Walsh functions, IEE Proc. Pt. D, pp. 89-92, (1991).

[6] C. F. Chen, C. H. Hsiao, Haar wavelet method for solving lumped and distributed-parameter systems, IEE Proc. Pt. D, Vol. 144, pp. 87-94, (1997).

[7] C. H. Hsiao, State analysis of linear time delayed systems via Haar wavelets, Math. Comput. Simul., 44, pp. 457-470, (1997).

[8] C. H. Hsiao, W.J. Wang, State analysis and optimal control of linear time- varying systems via Haar wavelets, Optim contr appl met. 19, pp. 423-433, (1998). 
[9] S. Sekar, K. Prabakaran, Numerical Solution of higher order Linear Singular System Using Single term Haar Wavelet series method, Int. J. of Mathematical Sciences and Applications, 1, pp. 737-746, (2011).

[10] V. Murugesh, K. Batri, State Analysis of Time-Varying Singular Bilinear Systems by RK-Butcher Algorithms, International Journal of Computers, Communications and Control, 3, pp. 103-109, (2008).

[11] B. Sepehriand, M. Razzaghi, Solution of time varying singular nonlinear systems by single term Walsh series, Math. Prob. Eng. 3, pp. 129-136, (2003).

[12] M. Garg, L. Dewan, A Generalized approach for state Analysis and parameter estimation of bilinear systems using Haar connection coefficients, World Academy of Science, Engineering and Technology 5, pp. 1094-1099, (2011).

[13] P. L. Liu, Further results on the stability analysis of singular systems with time-varying delay: A delay decomposition approach, International Journal of Analysis, ID 721407, pp. 1-11, (2013).

[14] H. Sandberg, A. Rantzer, Balanced Truncation of Linear Time-Varying Systems, IEEE Transactions on automatic control, 49, pp. 217-229, (2004).

[15] L. Zhou, C. Yang, Q. Zhang, Y. Lai, Finite-time stability analysis for linear time-varying singular impulsive systems, Scientific Research and Essays, 6, pp. 3344-3350, (2011).

[16] Mirmomeni, M., M. Shafiee, State analysis of time-invariant singular systems via Haar wavelet, 13th Iranian Conference on Electrical Engineering (ICEE 05), Zanjan University, Zanjan, Iran, 2005b.

[17] O. Nilsson, A. Rantzer, A novel approach to balanced truncation of nonlinear systems, European Control Conference, (2009).

[18] K. D. Mease, S. Bharadwaj, S. Iravanchy, Timescale analysis for nonlinear dynamical Systems, Journal of guidance, control, and dynamics, 26, pp. 318-330, (2003).

[19] M. Behroozifar, S. A. Yousefi, A. Ranjbar N, Numerical solution of optimal control of time-varying singular systems via operational matrices, International Journal of Engineering, 27, pp. 523-532, (2014). 
80 S. Raja B., S. G. Venkatesh, S. K. Ayyaswamy ES S. Balachandran

[20] K. Balachandran, K. Murugesan, Analysis of Electronic circuits using single term Walsh series approach , Int. J. Electron. 69, pp. 327-332, (1990).

[21] K. Balachandran, K. Murugesan, Analysis of transistor circuits using the single term Walsh series technique ,International Journal of Electronics, 71, pp. 397-401, (1991).

[22] M. Razzagi, S. Yousefi, Legendre wavelets method for the solution of nonlinear problems in the calculus of variations, Math. Comput. Modell., 34, pp. 45-54, (2001).

[23] S. Yousefi, M.Razzagi, Legendre wavelets method for the nonlinear Volterra-Fredholm integral equations, Math. Comput. Simul, 70, pp. $1-8,(2005)$.

[24] S. A .Yousefi, Legendre scaling function for solving generalized EmdenFowler equation, Int. J. Inf. Sys. Sci. 3, pp. 243-250, (2007).

[25] S. A .Yousefi, Legendre wavelets method for solving differential equations of Lane-emden type, Appl. Math. Comput., 181, pp. 1417-1422, (2006).

[26] S.G.Venkatesh, S.K. Ayyaswamy, S. Raja Balachandar, The Legendre wavelet method for solving initial value problems of Bratu-type, Comput. Math. Appl., 63, pp. 1287-1295, (2012).

[27] S. G. Venkatesh, S. K. Ayyaswamy, S. Raja Balachandar, K. Kannan, Convergence Analysis of Legendre wavelets method for solving Fredholm integral equations, Appl. Math. Sci., 6, pp. 2289-2296, (2012).

[28] S. G. Venkatesh, S.K. Ayyaswamy, S. Raja Balachandar, Legendre approximation solution for a class of higher-order Volterra integrodifferential equations, Ain Shams Eng J, 3, pp. 417-422, (2012).

[29] S. G. Venkatesh, S.K. Ayyaswamy, S. Raja Balachandar, Legendre wavelets based approximation method for Cauchy problems, Appl. Math. Sci. 6, pp. 6281-6286, (2012).

[30] S. G. Venkatesh, S. K. Ayyaswamy, S. Raja Balachandar, Legendre Wavelets based approximation method for solving advection problems, Ain Shams Eng J, 4, pp. 925-932, (2013). 
[31] S. G. Venkatesh, S. K. Ayyaswamy, S. Raja Balachandar, K. Kannan, Wavelet Solution for Class of Nonlinear Integro-diferential Equations, Indian Journal of Science and Technology, pp. 4670-4677, (2013).

[32] S. G. Venkatesh, S. K. Ayyaswamy, S. Raja Balachandar, K.Kannan, Legendre Wavelet Method for the Singular System of Transistor Circuits, International Journal of Applied Engineering Research, 9, pp. 213-221, (2014).

[33] M. Razzagi, S. Yousefi, The Legendre Wavelet operational matrix of integration, Int. J. Syst. Sci., 32 (4), pp. 495-502, (2001).

[34] P. A. Regalia, S. K. Mitra, Kronecker product, Unitary matrices and signal Processing applications, SIAM , 31, pp. 586-613, (1989).

[35] C. H. Hsiao, W. J. Wang, State analysis of time varying singular nonlinear systems via Haar wavelets, Math. Comput. Simul, 51, pp. 91100, (1999).

\section{S. Raja Balachandar}

Department of Mathematics

School of Humanities and Sciences

Sastra University,

Thanjavur-613 401,

Tamilnadu,

India

e-mail : srbala09@gmail.com

\section{S. G. Venkatesh}

Department of Mathematics

School of Humanities and Sciences

Sastra University,

Thanjavur-613 401,

Tamilnadu,

India

e-mail : venkamaths@gmail.com 
82 S. Raja B., S. G. Venkatesh, S. K. Ayyaswamy \& S. Balachandran

S. K. Ayyaswamy

Department of Mathematics

School of Humanities and Sciences

Sastra University, Thanjavur-613 401, Tamilnadu,

India

e-mail : sjcayya@yahoo.co.in

and

\section{S. Balachandran}

Department of Mathematics

School of Humanities and Sciences

SASTRA University,

Thanjavur-613 401,

Tamilnadu,

India

e-mail : bala@maths.sastra.edu 
Table 5.1: Comparison between Haar and Legendre wavelets solution for problem 2

\begin{tabular}{|c|c|c|c|c|c|c|c|c|c|c|}
\hline Time & \multicolumn{5}{|c|}{ Haar solution $\mathrm{m}=64[35]$} & \multicolumn{3}{c|}{ Legendre Wavelet solution } \\
\hline & $x_{1}(t)$ & $x_{2}(t)$ & $x_{3}(t)$ & $x_{4}(t)$ & $x_{5}(t)$ & $x_{1}(t)$ & $x_{2}(t)$ & $x_{3}(t)$ & $x_{4}(t)$ & $x_{5}(t)$ \\
\hline 0 & 0.0000 & 0.0000 & 1.0000 & 0 & 0.0000 & 0.0000 & 0.0000 & 1.0000 & 0 & 0.0000 \\
0.5 & -0.3297 & 0.5768 & 0.5683 & 0 & 0.1425 & -0.3299 & 0.5766 & 0.5682 & 0 & 0.1427 \\
1.0 & -0.4776 & 0.7999 & 0.4608 & 0 & 0.2578 & -0.4774 & 0.7998 & 0.4609 & 0 & 0.2577 \\
1.5 & -0.5486 & 0.9019 & 0.4198 & 0 & 0.3186 & -0.5485 & 0.9019 & 0.4197 & 0 & 0.3185 \\
2.0 & -0.5835 & 0.9510 & 0.4017 & 0 & 0.3495 & -0.5834 & 0.9511 & 0.4015 & 0 & 0.3496 \\
2.5 & -0.6008 & 0.9751 & 0.3931 & 0 & 0.3650 & -0.6006 & 0.9750 & 0.3930 & 0 & 0.3651 \\
3.0 & -0.6094 & 0.9871 & 0.3889 & 0 & 0.3728 & -0.6096 & 0.9870 & 0.3887 & 0 & 0.3727 \\
3.5 & -0.6137 & 0.9931 & 0.3869 & 0 & 0.3767 & -0.6135 & 0.9932 & 0.3868 & 0 & 0.3766 \\
4.0 & -0.6159 & 0.9961 & 0.3858 & 0 & 0.3787 & -0.6157 & 0.9960 & 0.3859 & 0 & 0.3788 \\
\hline & \multicolumn{10}{|c|}{}
\end{tabular}

Table 5.2: Comparison between Haar and Legendre wavelets solution for problem 3

\begin{tabular}{|c|c|c|c|c|c|c|}
\hline Time & \multicolumn{3}{|c|}{ Haar solution $\mathrm{m}=64[35]$} & \multicolumn{3}{c|}{ Legendre Wavelet solution } \\
\hline & $x_{1}(t)$ & $x_{2}(t)$ & $x_{3}(t)$ & $x_{1}(t)$ & $x_{2}(t)$ & $x_{3}(t)$ \\
\hline 0 & 2.000 & -1.0000 & -2.0000 & 2.000 & -1.0000 & -2.0000 \\
0.125 & 1.7517 & -0.7517 & -1.9719 & 1.7516 & -0.7516 & -1.9718 \\
0.250 & 1.5071 & -0.5071 & -1.9431 & 1.5072 & -0.5072 & -1.9430 \\
0.375 & 1.2660 & -0.2660 & -1.9135 & 1.2661 & -0.2662 & -1.9134 \\
0.5 & 1.0287 & -0.0287 & -1.8832 & 1.0288 & -0.0289 & -1.8833 \\
0.625 & 0.7953 & 0.2047 & -1.8519 & 0.7952 & 0.2048 & -1.8518 \\
0.75 & 0.5658 & 0.4342 & -1.8196 & 0.5657 & 0.4341 & -1.8195 \\
0.875 & 0.3404 & 0.6596 & -1.7862 & 0.3403 & 0.6595 & -1.7861 \\
1.0 & 0.1193 & 0.8807 & -1.7516 & 0.1194 & 0.8808 & -1.7512 \\
\hline & \multicolumn{7}{|c|}{} \\
\end{tabular}

